\title{
EARLY DUE LOW UNCERTAINTY (EDLU)FOR IMPROVING SUPPLY CHAIN PERFORMANCE UNDER ORDER VARIABILITY IN PRECAST CONCRETE PRODUCTION
}

\author{
Taehoon Kim ${ }^{1}$ and Yong-Woo Kim ${ }^{2}$
}

\begin{abstract}
The AEC (architect, engineering, and construction) industry finds a trend that more projects are adopting a prefabrication for various reasons. In a context of prefabrication, reliable supply chain is one of critical factors for project success. One of prefabricated products being adopted in building construction is precast concrete. A precast conctete supplier needs to optimize his production schedule while meeting various demands from multiple customers (i.e., contractors on project site). Most suppliers rely on dispatching rule in their production scheduling. However, contractor's order variability makes an impact on a supplier's production schedule and the reliability of supply chain. The authors proposed a new dispatching rule (EDLU, early due low uncertainty)taking into account a contractor's order reliability, followed by simulation experiments. The study suggests that (1) order variability leads to variance of prefabricated product delivery; (2) EDLU is more effective than traditional dispatching rules when order variability increases; (3) a proposed dispatching rule of EDLU gives incentives to a contractor's reliable order by giving production priority to orders with low uncertainty.
\end{abstract}

\section{KEYWORDS}

Precast concrete, production schedule, dispatching rule, EDLU (early due low uncertainty), operational strategy.

\section{INTRODUCTION}

The AEC (architect, engineering, and construction) industry finds a trend that more projects are adopting a prefabrication for various reasons. A study carried out by McGraw Hill Construction (2011) showed that nearly all construction stakeholders expect to utilize prefabrication in some of their projects. As a result, the effective management of prefabrication supply chain can make a considerably influence on the performances of construction projects. In a context of prefabrication, reliable supply chain is one of critical factors for project success.

1 Assistant Professor, Architectural Engineering Dept., Seoul National University of Science and Technology, Seoul, South Korea, kimth0930@korea.ac.kr, orcid.org/0000-0003-1637-7753

2 Professor, Construction Management Dept., University of Washington, Seattle, WA 98195, USA, +1 206616 1916, yongkim@uw.edu, orcid.org/0000-0001-7020-0700 
In projects using precast concrete structures (e.g., precast wall panels), precast concrete structures are manufactured off-site in a controlled environment, transported to the site, and lifted into place (Benjaoran et al. 2005). A precast conctete supplier needs to optimize his production schedule while meeting various demands from multiple customers (i.e., contractors on project site). Most suppliers do not have capital enough to invest advanved optimization scheduling tool to develop and update their production schedule. Accordingly, they rely on dispatching rule (i.e., the way to prioritize work orders) in their production scheduling to meet various demands (Kim et al. 2020).

However, contractor's order variability makes an impact on a supplier's production schedule and the reliability of supply chain. The authors proposed a new dispatching rule taking into account a contractor's order reliability, followed by simulation experiments. The authors finally proposes three operational strategies based on the simulation results.

\section{PRECAST CONCTETE SCHEDULING AND ORDER RELIABILITY}

\section{Precast Concrete Production Schedule}

Precast concrete production is a flow production system which consists of six processes: formwork assembling (m1), rebar and other all embedded parts installation ( $\mathrm{m} 2)$, concrete casting (m3), concrete curing (m4), formwork dismantling (m5), and PC product finishing (m6). The precast concrete production system can be classified into interruptabile production and uninterruptable production (Wang and $\mathrm{Hu}$ 2017). Among six processes, concrete curing (m4) is categorized as a parallel process because it doesn't need any external resources once concrete casting (m3) is complete (Kim et al 2020).

Literature on precast concrete production schedule is found. However, most studies have been carried out on precast conceret production scheduling without demand uncertainty considered. Dawood (1995) carried out the heuristic approach-based production schedule model for the precast concete. Benjaoran et al. (2005) suggested a production scheduling model using the genetic algorithm (GA) for bespoke precast concete with multiple molds. Yang et al. (2016) suggested the searching technique based on a multi-objective GA for evaluating the time and cost from production to assembly.

The authors also found that several studies carried out the scheduling problem for the precast concrete production under the uncertainty. Chan and Wee (2003) used GA to develop the heuristic approach based-schedule repair model to resolve schedule disturbance. However, they didn't include due date changes as uncertainty. Ko (2010) suggested the prnciples for schedule adjustment to cope with the demand variability. Ma et al. (2018) suggested an approach to optimize the rescheduling of multiple production lines for the PC to cope with production emergencies, but they didn't take count into the uncertainty of on-site schedule. Ho (2019) investigated the optimization using interprogramming under demand uncertainty and work station capacity of the supplier. Kim et al. (2020) proposed a simulation module for scheduling PC under due date changes, but they didn't focus on how the plan reliability can make an impact on supply chain performance.

\section{ORDER RELIABILITY}

A construction project schedule has some uncertainty that makes an impact on the activities related to installation of prefabricated products (Chan and Wee 2003). Planning reliability is directly related to the order of prefabricated products. Order variability (i.e., 
changes in delivery order) may come from (1) working out of optimum sequence", or (2) expediting or delaying the progress with pre-arranged sequence (Ballard and Arbulu 2004). Although order variability may also come from design changes, this study exclude this case because delivery orders are usually made only after shop drawings are approved. It is rare that product design changes after its shop drawing is approved.

The changes either in work sequence or in timing of the installers schedule frequently lead to changes in delivery order unless the installer has space enough to hold inventory. The changes in delivery order made by installers (i.e., contractors on site) disrupts the fabricator's production schedule, leading to additional costs and time. The delivery order changes (i.e. order variability) may occur before or during corresponding precast concrete production. If the delivery order changes prior to precast concrete production begins, the suppler should rearrange their production schedule. If the delivery order changes while precast concrete production in process, either the suppler or the contractor should hold the inventory unless the third party dealer who is in charge of logistics takes care of inventory.

\section{A SuPPlier's Produciton Schedule and DisPatching Rule}

There are multiple areas to respond to such order variability to reduce the negative impact on supply chain performances (i.e., lead time and costs). They include improving a contractor's planning reliability through the Last Planner System, setting up a production layout so that the supplier's production schedule can be flexible enough to respond to order changes, or having a contractor purchase the supplier's production capacity rather than products. The study focuses only on the supplier's production scheduling with the following assumption:

- A contractor's order reliability is given

- A production layout does not change. (i.e., the production duration is given)

- A contractor does not have any strategic solution to change the contractual relationship.

Many construction fabricators have limited planning capacity not enough to develop a robust scheduling or schedule optimization responding to order variability (Kim et al. 2020). Instead of complex scheduling method such as optimization algorithm, many construction fabricators have used dispatching rules in practice because of their simplicity and intuitiveness. The following is the list of dispatching rules being commonly used by the manufacturers.

- The EDD (earliest due date) rule has been widely used for production scheduling problem because of its simplity and better performance than other rules (Chan and $\mathrm{Hu}$ 2002). The EDD rule chooses the next job having earliest due date from the queue. This rule focuses on satisfying job due dates.

- The SPT (shortest processing time) rule chooses the next job having the shortest processing times from the queue. This rule has been known to be one of the best to reduce work-in-process inventory because the rule minimize the time a job stays in the shop (Weng and Ren 2006).

- The CR(critical ratio) rule chooses the next job considering the available time divided by the total remaining process time of the job.

The existing rules didn't take into account the order uncertainty which may change due dates of orders. This study propose a new rule of EDLU (early due and low uncertainty) 
taking into account. The PC production schedule can be more flexible by responding to order variability (i.e, the due date changes occur before the production gets started). However, it is strenuous to adjust the PC production schedule if the orders' due date changes are notified after the production gets started. The authors propose to shift the risk of production disruption to the party who creates the order variability (i.e, contractor who frequently changes the delivery order). Therefore, it was required that order with high uncertainty of the due date is started late among orders with a similar priority.

The proposed dispatching rule uses EDD as a baseline because EDD has been popularly applied for PCs production scheduling because it has better performance compared to other dispatching rules (Ho 2018). The proposed rule evaluates the due date and the contractor's order uncertainty when the order's due date is confirmed. The proposed one evaluates the due dates giving priority to the order with early due date in their production sequence. If multiple orders have the same due date, the proposed rule make the priority of orders having the higher uncertainty lower (Kim et al 2020).

\section{SIMULATION EXPERIMENT}

\section{Simulation Method}

Simulation experiments have been conducted to compare the performance of the proposed rule to existing rules such as EDD, SPT, and CR and verify the validity. For simulation experiments, the study set up several parameters such as the due date interval, due date tightness, due date uncertainty. In order to examine the effectiveness of reliability in supply lead time, the DPPSM (Dynamic Prefabricated Product Scheduling Model, Kim et al 2020), which has been developed for Precast Concrete Schedule Simulation, is adopted and simulated with the diverse cases.

The DPPSM uses a discrete-time simulation (DTS) method to model precast concrete production process. The DPPSM consists of two parts: (1) a due date uncertainty generator and (2) production scheduling system (Figure 1, Kim et al 2020). The due date uncertainty generator creates due date changes resulting from a predefined probability distribution function. The production scheduling system consists of a module of 'search and update' and 'priority evaluation.' If a priority rule is given, the module identifies a priority task. The DPPSM allows to reschedule the precast concrete production to cope with the due date changes. 


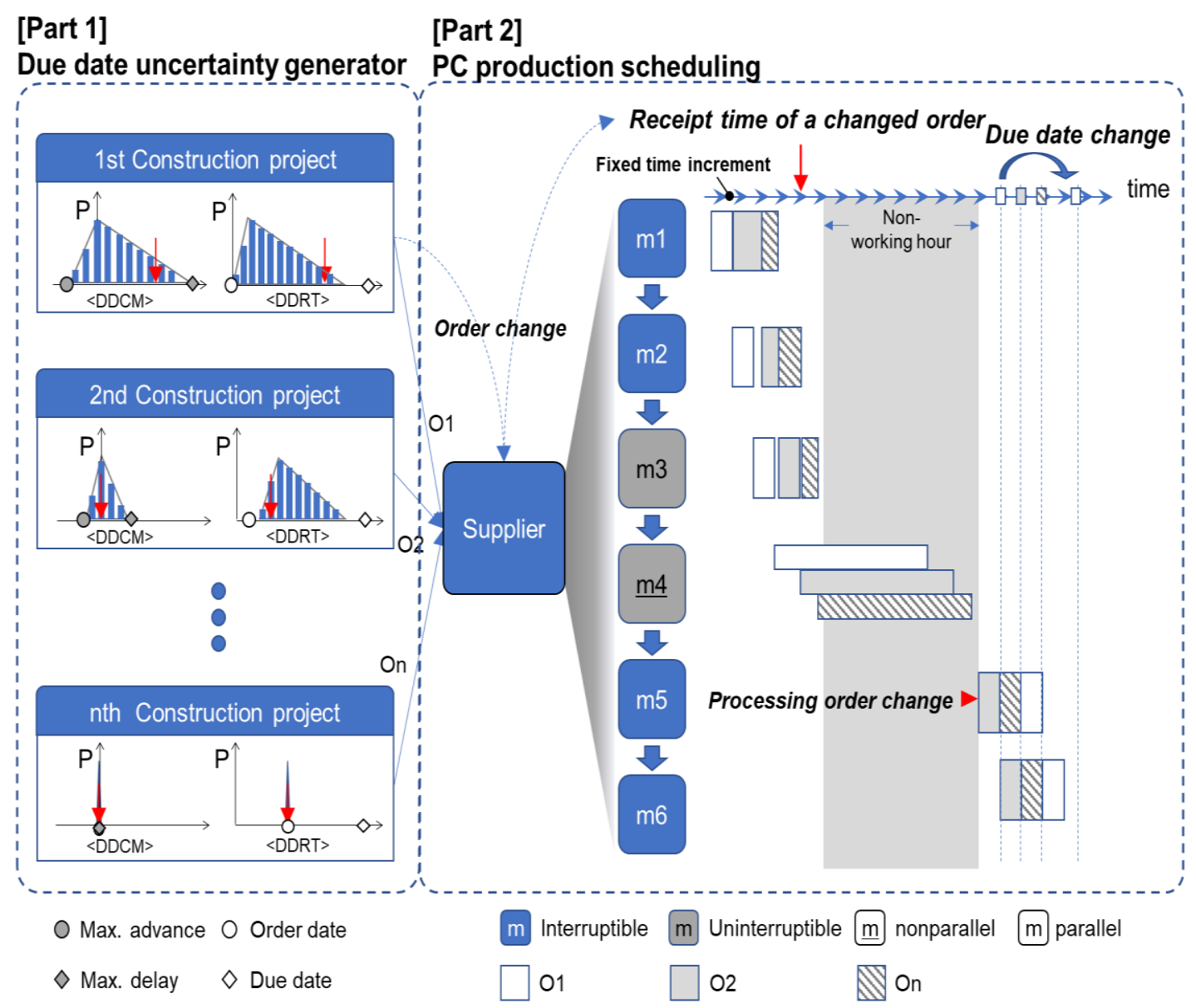

$\mathrm{P}$ indicates possibility; $\mathrm{m}$ indicates machine; $\mathrm{O}$ indicates order; DDCM indicates due date change magnitude; and DDRT indicates changed due date receipt time.

Figure 1: DPPSM (Dynamic Prefabricated Product Scheduling Model, Kim et al 2020)

\section{SIMULATION EXPERIMENTS}

\section{Description of Scenarios}

The simulation experiments in this study assumed several conditions as scenarios (Table 1). There were total fifteen orders. The all order dates were Day 1 and their due times were the end time on the due date. The receipt time about the changed due date was the start time on the due date. The original due date (ODD) classified into three types: order date $(\mathrm{OD})+\mathrm{t}$. $\mathrm{OD}+\mathrm{t}+\mathrm{a}, \mathrm{OD}+\mathrm{t}+2 \mathrm{a}$. The variable $\mathrm{t}$ indicates the due date tightness level. The variable $a$ is related to the gap day between two jobs' due dates and indicates the production load level. 
Table 1. PCs Order Information in Simulation Experiments.

\begin{tabular}{|c|c|c|c|c|c|c|}
\hline \multirow{2}{*}{ No. } & \multirow{2}{*}{ Job } & \multirow{2}{*}{ Product type } & \multirow{2}{*}{ OD } & \multirow{2}{*}{ ODD } & \multicolumn{2}{|c|}{ Uncertainty } \\
\hline & & & & & Min. Due date & Max. Due date \\
\hline 1 & J1 & $\mathrm{P} 1$ & Day 1 & $O D+t$ & ODD -1 & ODD + u \\
\hline 2 & & $\mathrm{P} 2$ & & $O D+t$ & ODD -1 & ODD + u \\
\hline 3 & & P3 & & $O D+t+a$ & ODD -1 & ODD + u \\
\hline 4 & & P5 & & $O D+t+a$ & ODD -1 & ODD + u \\
\hline 5 & & P7 & & $O D+t+2 a$ & ODD -1 & ODD + u \\
\hline 6 & J2 & $\mathrm{P} 2$ & Day 1 & $O D+t$ & ODD -1 & ODD + u \\
\hline 7 & & $\mathrm{P} 4$ & & $O D+t$ & ODD -1 & ODD + u \\
\hline 8 & & P8 & & $O D+t+a$ & ODD -1 & ODD $+u$ \\
\hline 9 & & P9 & & $O D+t+a$ & ODD -1 & ODD + u \\
\hline 10 & & P10 & & $O D+t+2 a$ & ODD -1 & ODD $+u$ \\
\hline 11 & J3 & P2 & Day 1 & $O D+t$ & ODD -1 & ODD $+u$ \\
\hline 12 & & P4 & & $O D+t$ & ODD -1 & ODD $+u$ \\
\hline 13 & & P6 & & $O D+t+a$ & ODD -1 & ODD $+u$ \\
\hline 14 & & P8 & & $O D+t+a$ & ODD -1 & ODD $+u$ \\
\hline 15 & & P10 & & $O D+t+2 a$ & ODD -1 & ODD + u \\
\hline
\end{tabular}

In terms of uncertainty, all jobs had the same due dates variance. The maximum delay of and a maximum advance of All jobs were $u$ and one days, respectively; The focus of this study placed the maximum delay because construction delays happens more frequently than early construction completion (Kim et al. 2020).

The processing time and mold type in this study are shown in Table 2 (Benjaoran et al 2005). This study assumed the quaitity of each mold type is two molds to consider resource constraint in the real PCs production situations. The simulation time advance step unit for DPPSM was set to 0.1 hour.

Table 2. Processing Time for Each Machine and Mold Type according to Product Type

\begin{tabular}{cccccccc}
\hline $\begin{array}{c}\text { Product } \\
\text { type }\end{array}$ & Mold type & \multicolumn{7}{c}{ Processing time (h) } \\
\hline P1 & A & 2.0 & 1.6 & 2.4 & 12.0 & 2.5 & 1.0 \\
P2 & B & 3.4 & 4.0 & 4.0 & 12.0 & 2.4 & 5.0 \\
P3 & A & 0.8 & 1.0 & 1.2 & 12.0 & 0.8 & 0.1 \\
P4 & A & 0.6 & 0.8 & 1.0 & 12.0 & 0.6 & 2.0 \\
P5 & C & 3.0 & 3.6 & 2.4 & 12.0 & 2.4 & 3.0 \\
P6 & A & 3.0 & 3.2 & 3.0 & 12.0 & 3.0 & 1.6 \\
P7 & C & 1.3 & 0.9 & 2.4 & 12.0 & 1.9 & 1.8 \\
P8 & B & 1.7 & 1.4 & 1.1 & 12.0 & 0.9 & 0.7 \\
P9 & A & 2.2 & 1.8 & 1.2 & 12.0 & 2.3 & 0.7 \\
P10 & C & 1.6 & 3.2 & 2.3 & 12.0 & 2.1 & 2.7
\end{tabular}

Note: $\mathrm{m} 1$, mold assembling; $\mathrm{m} 2$, reinforcement and placing of all embedded parts; $\mathrm{m} 3$, concrete casting; $\mathrm{m} 4$, concrete curing; $\mathrm{m} 5$, mold dismantling; $\mathrm{m} 6$, product finishing, 
The authors simulated a total of 18 scenarios. The daily working hours was assumed to ten hours. The variable $u$ which means the due date uncertainty differed from one days to five days with two-day gap. The variable $t$ which means the due date tightness differed from one day to three days with one-day gap, and the variable $a$ which means production load level differd from one day to three days with a two-day gap.

\section{Simulation Results}

The authors showed the relative performance by calculating the increase in terms of total tardiness using each rule with compared to the proposed rule. As a result of simulating all scenarios, the average relative performance over the 300 replications are shown in Table 3. The scenarios were named as 'Suta'. For example, the S321 means a scenario with three of $u$, two of $t$, and one of $a$.

Table 3. Simulation Results of Dispatching Rules

\begin{tabular}{ccccc}
\hline Scenario & DPPSM & CR & EDD & SPT \\
\hline S111 & $742.4(0,0 \%)$ & $699.2(-43.2,-5.8 \%)$ & $723.8(-18.5,-2.5 \%)$ & $490.7(-251.7,-33.9 \%)$ \\
S113 & $331.4(0,0 \%)$ & $316.2(-15.2,-4.6 \%)$ & $330.2(-1.2,-0.4 \%)$ & $364.6(33.2,10 \%)$ \\
S121 & $365.0(0,0 \%)$ & $407.1(42.1,11.5 \%)$ & $384.1(19.1,5.2 \%)$ & $311.5(-53.5,-14.7 \%)$ \\
S123 & $131.2(0,0 \%)$ & $138.1(6.9,5.3 \%)$ & $132.4(1.2,0.9 \%)$ & $232.9(101.7,77.5 \%)$ \\
S131 & $152.0(0,0 \%)$ & $158.3(6.3,4.2 \%)$ & $154.0(2,1.3 \%)$ & $173.1(21.1,13.9 \%)$ \\
S133 & $31.4(0,0 \%)$ & $39.4(8.0,25.5 \%)$ & $32.3(0.9,3 \%)$ & $136.0(104.6,333.2 \%)$ \\
\hline S311 & $407.6(0,0 \%)$ & $417.9(10.4,2.5 \%)$ & $417.7(10.1,2.5 \%)$ & $337.9(-69.7,-17.1 \%)$ \\
S313 & $158.3(0,0 \%)$ & $167.4(9.1,5.7 \%)$ & $165.4(7.1,4.5 \%)$ & $249.8(91.4,57.8 \%)$ \\
S321 & $178.4(0,0 \%)$ & $190.4(12.0,6.7 \%)$ & $182.6(4.3,2.4 \%)$ & $190.3(11.9,6.7 \%)$ \\
S323 & $56.4(0,0 \%)$ & $61.1(4.6,8.2 \%)$ & $59.5(3.1,5.4 \%)$ & $148.8(92.4,163.6 \%)$ \\
S331 & $51.2(0,0 \%)$ & $60.5(9.3,18.2 \%)$ & $52.1(1.0,1.9 \%)$ & $101.4(50.2,98.1 \%)$ \\
S333 & $12.3(0,0 \%)$ & $17.7(5.4,44.2 \%)$ & $13.3(1.0,7.8 \%)$ & $82.1(69.8,567.8 \%)$ \\
\hline S511 & $218.1(0,0 \%)$ & $232.2(14.2,6.5 \%)$ & $226.8(8.7,4.0 \%)$ & $226.7(8.7,4 \%)$ \\
S513 & $87.1(0,0 \%)$ & $93.1(6.0,6.9 \%)$ & $96.3(9.2,10.6 \%)$ & $172.1(85.0,97.6 \%)$ \\
S521 & $88.2(0,0 \%)$ & $103.1(14.9,16.9 \%)$ & $94.1(5.9,6.7 \%)$ & $128.4(40.3,45.7 \%)$ \\
S523 & $31.7(0,0 \%)$ & $36.9(5.2,16.2 \%)$ & $36(4.2,13.3 \%)$ & $98.3(66.6,209.8 \%)$ \\
S531 & $25.5(0,0 \%)$ & $27.2(1.6,6.3 \%)$ & $26.2(0.7,2.7 \%)$ & $62.2(36.6,143.4 \%)$ \\
S533 & $9.0(0,0 \%)$ & $9.8(0.8,9.2 \%)$ & $9.2(0.2,2.2 \%)$ & $51.9(42.9,476.4 \%)$ \\
\hline Tw0 & & $1 n$ & &
\end{tabular}

Two numbers in parentheses indicate increase and ratio compared to DPPSM. For example, two numbers in parentheses of $\mathrm{CR}$ in $\mathrm{S} 111$ were calculated by $-43.2=$ $699.2-742.4,-5.8 \%=-43.2 / 742.4$.

The DPPSM showed better performance in most of scenarios with three $(\mathrm{u}=1)$, five $(\mathrm{u}=3)$, and six $(\mathrm{u}=5)$. Also, the DPPSM made better performance as $\mathrm{u}$ increases compared to the other rules. In case of scenarios with tight due date such as S111 and S311, SPT was the best rule showing the lowest tardiness, which was as known (Weng and Ren 2006). These results show that the DPPSM tends to be superior to using the existing rules as the due date uncertainty increases. 


\section{DISCUSSIONS AND CONCLUSIONS}

In this simulation experiments, the authors tested four different dispatching rules (or priority rules) in job shop scheduling for precast concrete production when there exists order variability by a contractor. In most cases where order variability, the simulation results suggest that a new priority rule of EDLU, which penalizes a job order by a contractor with low order reliability, shows better delivery performances in terms of the average lead time and its variance. In light of lean construction principles, the simulation results suggest the followings:

First, order variability leads to variance of prefabricated product delivery. The best way to reduce order variability is to improve a contractor's planning reliability. The lean construction literature has shown that the planning reliability makes an impact on project schedule and productivity of trades on sites. The simulation experiments suggest that the order variability makes a negative impact on the lead time and its variance of prefabricated products.

Second, EDLU is more effective than traditional dispatching rules when order variability increases. The paper proposes a new dispatching rule of EDLU. The proposed EDLU may help the precast concrete suppliers develop their job shop schedule when there is order variability.

This study supposed that the due date uncertainty has the uniform distribution. The authors will conduct the further study to verify the effectiveness of the proposed model with the distribution shape of the uncertainty obtained from real construction projects.

\section{REFERENCES}

Ballard, G. and Arbulu, R. (2004). "Making Prefabrication Lean”, International Group of Lean Construction, Helsingor, Denmark.

Benjaoran, V., Dawood, N., and Hobbs, B. (2005). "Flowshop scheduling model for bespoke precast concrete production planning". Construction Management and Economics, 23(1), 93-105.

Chan, W. T. and Hu, H. (2002). "Production scheduling for precast plants using a flow shop sequencing model". Journal of computing in civil engineering, 16(3), 165-174.

Chan, W. T. and Wee, T. H. (2003). "A Multi-Heuristic GA for Schedule Repair in Precast Plant Production". International Conference on Automated Planning and Scheduling, American Association for Artificial Intelligence, Trento, Italy, June 9-13 (pp.236-245).

Dawood, N. N. (1995). "Scheduling in the precast concrete industry using the simulation modelling approach". Building and Environment, 30(2), 197-207.

Ho, C. (2019). Application of optimization to the production planning of construction prefabrication supply chains. Ph.D. Dissertation, College of Built Environment, University of Washington.

Kim, J. M., Zhou, Y. D., \& Lee, D. H. (2017). "Priority scheduling to minimize the total tardiness for remanufacturing systems with flow-shop-type reprocessing lines". The International Journal of Advanced Manufacturing Technology, 91(9-12), 3697-3708.

Kim, T., Kim, Y., and Cho, H. (2020). "Dynamic production scheduling model under due date uncertainty in precast concrete construction", Journal of Cleaner Production, 257.

Ko, C. H. (2010). "Production control in precast fabrication: considering demand variability in production schedules". Canadian Journal of Civil Engineering, 38(2), 191-199. 
Ma, Z., Yang, Z., Liu, S., and Wu, S. (2018). "Optimized rescheduling of multiple production lines for flowshop production of reinforced precast concrete components". Automation in Construction, 95, 86-97.

McGraw Hill Construction. (2011). Prefabrication and Modularization: Increasing productivity in the construction industry. Smart Market Report, New York.

Wang, Z. and $\mathrm{Hu}, \mathrm{H}$. (2017). "Improved precast production-scheduling model considering the whole supply chain". Journal of computing in civil engineering, 31(4), 04017013.

Weng, M. X. and Ren, H. (2006). “An efficient priority rule for scheduling job shops to minimize mean tardiness". IIE transactions, 38(9), 789-795.

Yang, Z., Ma, Z. and Wu, S. (2016). "Optimized flowshop scheduling of multiple production lines for precast production". Automation in Construction, 72, 321-329. 